\title{
Tratamento térmico úmido em sementes de Acacia mangium
}

\section{Moist heat treatment on Acacia mangium seeds}

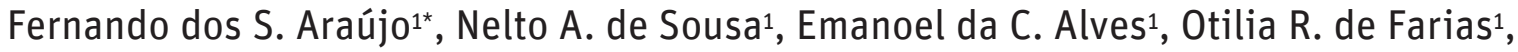

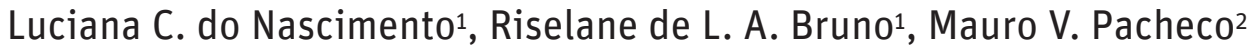

${ }^{1}$ Universidade Federal da Paraíba, Centro de Ciências Agrárias, Departamento de Fitotecnia e Ciências Ambientais. Rodovia PB 079, km 12, Caixa Postal 66, 58397-000, Areia, Paraíba, Brasil 2 Universidade Federal do Rio Grande do Norte. Unidade Acadêmica Especializada em Ciências Agrárias. Rodovia RN 160, km 3, Distrito de Jundiaí, Caixa postal 07, 59280-000, Macaíba, Rio Grande do Norte, Brasil

( ${ }^{\star}$ E-mail: nandosantos005@hotmail.com)

http://dx.doi.org/10.19084/RCA17023

Recebido/received: 2017.02.06

Recebido em versão revista/received in revised form: 2018.02.27

Aceite/accepted: 2018.03.01

\section{R E S U M O}

Acacia mangium Willd. é uma espécie arbórea, exótica, que possui potencial para exploração de produtos florestais madeireiros no Brasil. Como esta se propaga, principalmente, via sexuada torna-se relevante o tratamento fitossanitário das sementes destinadas à produção de mudas. Assim, este estudo objetivou avaliar os efeitos do tratamento térmico via calor úmido na qualidade sanitária e fisiológica de sementes de A. mangium. Para tanto, as sementes foram tratadas em água aquecida à temperatura de $70^{\circ} \mathrm{C}$ durante os períodos de exposição de $0,10,15$ e 20 minutos. Em seguida, as mesmas foram submetidas à análise sanitária e ao teste de germinação. Com base na análise sanitária os fungos dos gêneros Aspergillus sp., Penicillium sp., Pestalotia sp., Cladosporium sp., Alternaria sp., Fusarium sp. e Botrytis sp.estavam associados às sementes. $\mathrm{O}$ tratamento térmico com calor úmido à temperatura de $70^{\circ} \mathrm{C}$ durante 10 a 15 minutos de exposição superou a dormência e reduziu a ocorrência de fungos associados às sementes de A. mangium.

Palavras-chave: Acácia australiana, florestal, termoterapia, fungos.

\section{A B S T R A C T}

Acacia mangium Willd. is an arboreal and exotic species, which has potential for exploitation of timber products in Brazil. As it spreads mainly through sexual reproduction, it becomes relevant the phytosanitary treatment of seeds for the production of seedlings. Therefore, this study aimed to evaluate the effects of thermal treatment humid heat on health and physiological quality of $A$. mangium seeds. Thus, the seeds were thermal treated in water heated to $70^{\circ}$ C during 0, 10, 15 and 20 minutes of exposure. Then, the seeds were subjected to germination test and seed health. The seed health analysis revealed that fungi of the genre Aspergillus sp., Penicillium sp., Pestalotia sp., Cladosporium sp., Alternaria sp., Fusarium sp. and Botrytis sp., were associated with seeds. The thermal treatment with humid heat at a temperature of $70^{\circ} \mathrm{C}$ for 10 to 15 minutes of exposure overcame the physical dormancy of seeds of A. mangium and reduced occurrence of fungal species.

Keywords: Australian acacia, forest seeds, thermotherapy, fungi.

\section{INTRODUÇÃO}

As sementes podem abrigar e transportar microrganismos ou agentes patogênicos de todos os grupos taxonômicos, causadores e não causadores de doenças (Brasil, 2009), os quais podem ser reduzidos ou erradicados através de tratamentos químicos, físicos, biológicos e bioquímicos (Menten e Moraes, 2010).

O tratamento físico mais comum é o térmico, que consiste na exposição das sementes à ação do calor 
seco ou úmido (Braga et al., 2010), sendo a temperatura e tempo de exposição controlados, pois o calor pode prejudicar a qualidade fisiológica das sementes (Machado, 2000).

O tratamento térmico, também chamado de termoterapia, pode ser considerado uma opção em potencial para tratamento fitossanitário de sementes de espécies que não possuem princípios ativos químicos registrados. Em espécies florestais, esse tratamento tem demonstrado ser eficiente no controle da micoflora associada às sementes de espécies arbóreas como Amburana cearensis A.C. Smith (Oliveira et al., 2011) e Pinus radiata D. Don (Agustí-Brisach et al., 2012) e arbustivas como Eugenia brasiliensis Lam., Eugenia uniflora L. (Françoso e Barbedo, 2014) e Jatropha curcas L. (Schneider et al., 2015).

Considerando que as sementes são importantes veículos de disseminação e transmissão de doenças em plantas (Brasil, 2009), a escassez de conhecimentos acerca da micoflora associada a sementes de espécies florestais representa um risco à formação de novos plantios, sobretudo, daquelas cuja propagação é realizada principalmente por meio de sementes. Esta é uma preocupação oriunda dos riscos à biossegurança e também do impacto econômico sobre o comércio de mudas (McCartan et al., 2015).

Dentre as espécies florestais exóticas cultivadas no Brasil que ainda necessitam de estudos nesse sentido pode-se destacar a Acacia mangium Willd. Esta é uma espécie arbórea utilizada em plantios comerciais para exploração de produtos como celulose, madeira para movelaria, construção civil e como fonte energética (Galiana et al., 2002). Tais potencialidades sugerem que esta árvore tem grande potencial para exploração de produtos florestais madeireiros no Brasil. Dessa forma, justificam-se a realização de estudos que permitam identificar a microflora fúngica associada às suas sementes, bem como buscar métodos para o controle de fungos potencialmente patogênicos.

Assim, objetivou-se com este trabalho avaliar os efeitos do tratamento térmico, via calor úmido, na qualidade sanitária e fisiológica de sementes de $A$. mangium.

\section{MATERIAL E MÉTODOS}

\section{Obtenção do lote de sementes}

O lote de sementes de $A$. mangium utilizado neste estudo foi proveniente de um plantio experi-

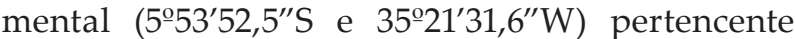
à Universidade Federal do Rio Grande do Norte (UFRN) no município de Macaíba, Estado do Rio Grande do Norte, Brasil. Os frutos foram coletados no início da deiscência natural em 15 indivíduos e encaminhados ao Laboratório de Sementes Florestais da UFRN para a extração manual e homogeneização das sementes. Em seguida, as mesmas foram acondicionadas em recipiente de vidro e armazenadas em refrigerador, com temperatura e umidade relativa médias de $5,0^{\circ} \mathrm{C}$ e $45 \%$ respectivamente.

Após 90 dias de armazenamento, as sementes foram enviadas ao Laboratório de Análise de Sementes do Centro de Ciências Agrárias da Universidade Federal da Paraíba (CCA-UFPB) no município de Areia, Estado da Paraíba, Brasil onde foram submetidas a uma seleção visual para exclusão de sementes deformadas e danificadas por insetos. Em seguida, foi realizada a determinação do grau de umidade pelo método da estufa (Brasil, 2009), utilizando-se duas subamostras de $26 \mathrm{~g}$ de semente. Na ocasião, as sementes apresentaram grau de umidade de $8,4 \%$.

\section{Aplicação dos tratamentos}

No Laboratório de Fitopatologia do CCA-UFPB, foram separadas do lote, amostras de 200 sementes, as quais foram acondicionadas em sacos de tecido perfurados e, imersos em água destilada mantida sob aquecimento em banho-maria regulado à temperatura de $70 \pm 3^{\circ} \mathrm{C}$, onde permaneceram expostas por 0, 10, 15 e 20 minutos. Após cada período de exposição, os sacos contendo as sementes foram retirados da água e abertos, sendo as sementes colocadas para secar naturalmente sobre papel toalha em condições de laboratório $\left(25 \pm 2^{\circ} \mathrm{C}\right)$.

Adotou-se um controle negativo (sementes sem tratamento) que foi representado pelo período de exposição de 0 minuto; e um controle positivo 
(tratamento fungicida padrão) no qual as sementes foram tratadas com fungicida de contato a base de captana $\left(\operatorname{Captan}^{\circledR}\right)$ na dosagem de 2,4 g.kg-1 de sementes.

\section{Avaliação da qualidade sanitária}

Para análise sanitária foi utilizado o método de incubação em substrato de papel conhecido como "Blotter Test" sugerido nas regras para análise sanitária de sementes adotadas no Brasil (Brasil, 2009). Para tanto, as sementes de cada tratamento foram dispostas individualmente sobre duas camadas de papel de filtro umedecido com água (destilada e esterilizada) e dispostas em placas de Petri de vidro transparente. As placas foram tampadas, vedadas com plástico filme e incubadas por dez dias em ambiente com temperatura média de $25 \pm 5^{\circ} \mathrm{C}$ e fotoperíodo de aproximadamente oito horas de luz natural indireta.

Após o período de incubação, as sementes foram examinadas individualmente com auxilio de um estereomicroscópio com resolução de 30-80X para identificação de estruturas de crescimento típicas de cada gênero fúngico. Para confirmação da etiologia fúngica foram preparadas lâminas para exame em microscópio ótico. Os resultados da ocorrência total e de cada gênero fúngico foram expressos em porcentagem.

\section{Avaliação da qualidade fisiológica}

Para o teste de germinação utilizou-se a metodologia sugerida pelas instruções para análise de sementes florestais adotada no Brasil (Brasil, 2013) que prescreve o substrato rolo de papel e a temperatura constante de $35^{\circ} \mathrm{C}$ para a espécie em estudo. Durante o teste, foi realizada a contabilização diária das sementes germinadas, cujos valores foram utilizados para calcular o índice de velocidade de germinação conforme Maguire (1962). No 21. dia após a instalação do teste foi computada a porcentagem de plântulas normais (sementes germinadas), anormais e sementes mortas conforme os critérios estabelecidos por Brasil (2009).

\section{Delineamento experimental e análise estatística}

O ensaio foi conduzido em delineamento inteiramente casualizado, utilizando-se 200 sementes subdivididas em quatro repetições para o teste de germinação e 200 sementes subdivididas em 20 repetições para a análise sanitária. Os dados que atenderam aos pressupostos para análise paramétrica foram submetidos à análise de regressão linear até segundo grau, selecionando-se o modelo significativo a $5 \%$ pelo teste $\mathrm{F}$ com maior coeficiente de determinação $\left(\mathrm{R}^{2}\right)$. Aqueles que não atenderam aos pressupostos para análise paramétrica foram analisados pelo teste não-paramétrico de KruskalWallis ao nível de 5\% de significância (Santana e Ranal, 2004). Essas análises foram realizadas utilizando-se o software estatístico Assistat (versão 7.7 beta) (Silva e Azevedo, 2002).

\section{RESULTADOS E DISCUSSÃO}

A análise sanitária das sementes de A. mangium revelou a ocorrência de fungos dos gêneros Aspergillus spp. (5\%), Penicillium sp. (4,5\%), Pestalotia sp. $(1,0 \%)$, Cladosporium sp. $(0,5 \%)$, Alternaria sp. $(0,5 \%)$, Fusarium sp. $(0,5 \%)$ e Botrytis sp. (0,5\%). Devido à baixa porcentagem de ocorrência, todos os gêneros fúngicos foram considerados como tendo baixo potencial de inóculo para as sementes de A. mangium (Quadro 1). Entretanto, justifica-se o controle de alguns deles como Fusarium sp. e Alternaria sp. que são potenciais causadores de infecções em sementes e plântulas de espécies florestais (Benetti et al., 2009; Lazarotto, et al., 2010; Maciel et al., 2012), assim como Aspergillus spp. que, apesar de não ser patogênico às plântulas, podem acelerar a deterioração das sementes durante o armazenamento de forma a reduzir a sua longevidade (Marchi et al. 2011; Barbosa et al., 2014).

Os resultados apresentados no Quadro 1 demonstram o efeito do tratamento térmico com calor úmido e do fungicida químico no controle dos fungos associados às sementes de $A$. mangium. Pode-se verificar que a porcentagem total de ocorrência dos fungos foi reduzida drasticamente quando as sementes foram tratadas com calor úmido por 10, 15 e 20 minutos de exposição e com fungicida químico, os quais não diferiram estatisticamente entre si. 
Quadro 1 - Porcentagem de ocorrência de gêneros fúngicos em sementes de Acacia mangium submetidas ao tratamento térmico úmido $\left(70^{\circ} \mathrm{C}\right)$ durante 0 (controle), 10, 15 e 20 minutos e tratamento químico antifúngico (Captana)

\begin{tabular}{|c|c|c|c|c|c|c|c|c|}
\hline Tratamento & $\begin{array}{c}\text { Aspergillus } \\
\text { spp. }\end{array}$ & $\begin{array}{l}\text { Penicillium } \\
\text { sp. }\end{array}$ & $\begin{array}{c}\text { Cladosporium } \\
\text { sp. }\end{array}$ & $\begin{array}{l}\text { Pestalotia } \\
\text { sp. }\end{array}$ & Alternaria sp. & $\begin{array}{c}\text { Fusarium } \\
\text { sp. }\end{array}$ & $\begin{array}{c}\text { Botrytis } \\
\text { sp. }\end{array}$ & Total \\
\hline Controle (0 min.) & 5,0 & 4,5 & 0,5 & 1,0 & 0,5 & 0,5 & 0,5 & $12,5 \mathrm{~b}$ \\
\hline $10 \mathrm{~min}$. & 1,5 & 0,0 & 0,5 & 0,0 & 0,0 & 0,0 & 0,0 & $2,0 \mathrm{a}$ \\
\hline $15 \mathrm{~min}$. & 0,5 & 0,5 & 0,0 & 0,0 & 0,0 & 0,0 & 0,0 & $1,0 \mathrm{a}$ \\
\hline $20 \mathrm{~min}$. & 0,5 & 0,0 & 0,0 & 0,0 & 0,0 & 0,5 & 0,0 & $1,0 \mathrm{a}$ \\
\hline Captana & 0,0 & 0,0 & 0,0 & 0,0 & 0,0 & 0,0 & 0,0 & $0,0 \mathrm{a}$ \\
\hline Valor-H & $7,79^{\text {ns }}$ & $9,22 \mathrm{~ns}$ & $4,75^{\text {ns }}$ & 8,00 ns & 8,00 ns & $6,13^{\mathrm{ns}}$ & $8,00^{\mathrm{ns}}$ & 34,98 \\
\hline
\end{tabular}

H-crítico = 9,48; ${ }^{\star}=$ significativo $(\mathrm{H}>\mathrm{H}$-crítico $)$ e ns = não significativo $(\mathrm{H}<\mathrm{H}$-crítico $)$ pelo teste de Kruskal-Wallis ao nível de $5 \%$ de probabilidade. Valores seguidos por letras distintas na coluna diferem estatisticamente entre si.

Estes resultados demonstraram que o tratamento térmico apresentou eficiência similar ao fungicida químico na redução da porcentagem de ocorrência total dos fungos associados às sementes de A. mangium. A utilização do maior tempo de exposição (20 minutos) promoveu a erradicação de Penicillium sp., Cladosporium sp., Pestalotia sp., Alternaria sp. e Botrytis sp., enquanto que Fusarium sp. e Aspergillus spp. só foram erradicados com fungicida químico, indicando que a temperatura e os períodos de exposição testados neste estudo foram insuficientes para eliminar completamente os inóculos desses agentes.

Ao analisar os resultados do teste de germinação (Figura 1) verifica-se que a porcentagem de germinação e a velocidade de germinação aumentaram gradualmente com a elevação do período de exposição ao calor úmido, mostrando que o mesmo agiu como um método de superação da dormência das sementes dessa espécie. Isto ocorreu, provavelmente, por que a água aquecida tem a capacidade de remover os elementos estruturais responsáveis pela impermeabilidade do tegumento à água (Baskin et al., 1998), permitindo às sementes absorver umidade, que é um dos requisitos básicos para que a germinação ocorra (Bewley et al., 2013).

De acordo com as curvas de regressão ajustadas ao modelo quadrático (Figura 1), verifica-se que o melhor desempenho germinativo (germinação e IVG) das sementes de $A$. mangium foi obtido entre
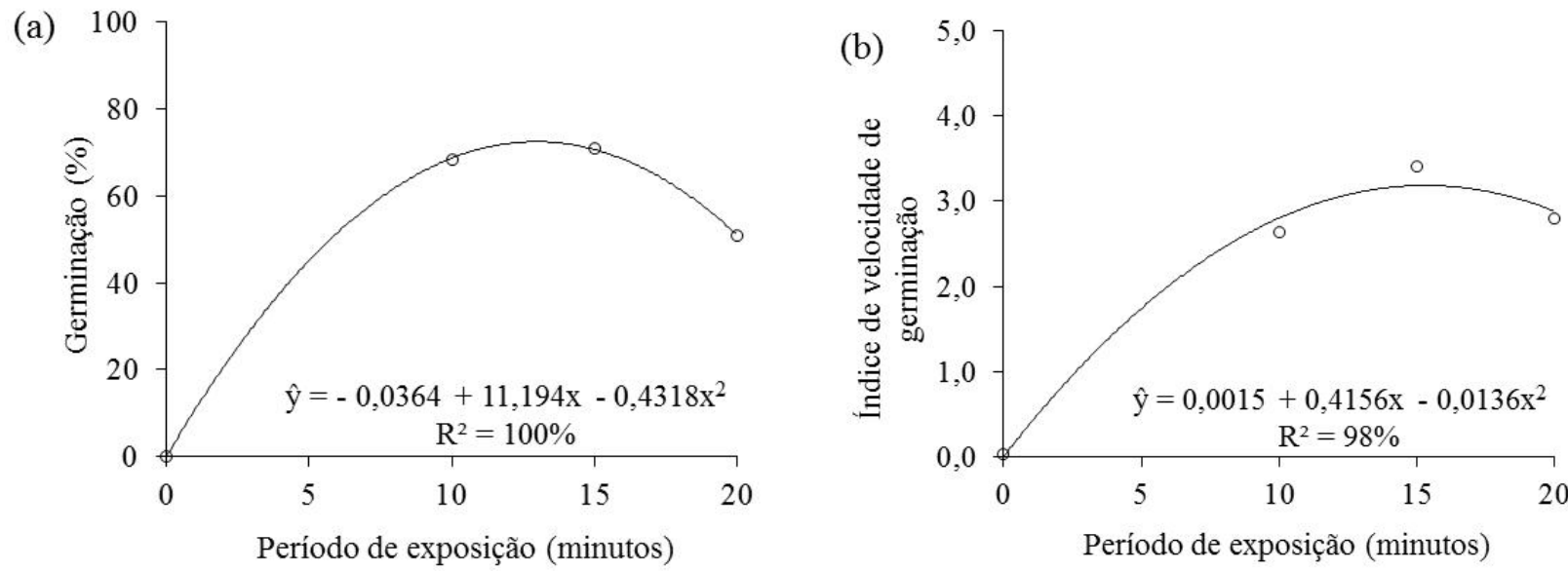

Figura 1 - Valores médios da porcentagem de germinação (a) e do índice de velocidade de germinação (b) de sementes de Acacia mangium submetidas ao tratamento térmico úmido $\left(70^{\circ} \mathrm{C}\right.$ ) durante 0 (controle), 10, 15 e 20 minutos. 
10 e 15 minutos de exposição ao calor úmido, pois as estimativas dos pontos de máxima eficiência física foram obtidas dentro dessa faixa.

O tratamento térmico com calor úmido também superou a dormência das sementes de outras espécies da família Fabaceae como Peltophorum dubium (Spreng.) Taub. (Lazarotto et al., 2013) e Dimorphandra mollis Benth. (Honório et al., 2010), contudo, não foi eficiente como método fungicida devido ao efeito negativo sobre a qualidade fisiológica das sementes. No caso de $p$. dubium o período de exposição necessário para controlar os fungos reduziu a qualidade fisiológica das sementes e o vigor das plântulas, enquanto para $D$. mollis o aumento na temperatura de tratamento deixou as sementes vulneráveis a infecções por fungos saprófitos provocando alta mortalidade de sementes. Esses resultados mostraram que não foi possível combinar a temperatura e o período de exposição necessário à eliminação dos patógenos sem prejudicar a qualidade fisiológica das sementes de forma a limitar a utilização dessa técnica para as sementes dessas espécies.

Apesar do aumento do período de exposição das sementes de A. mangium ao calor úmido ter favorecido a germinação e reduzido o percentual de ocorrência total dos fungos, verifica-se também que houve aumento da mortalidade de sementes (Figura 2a) e formação de plântulas anormais (Figura 2b).
Ao analisar as curvas de regressão ajustadas ao modelo linear (Figura 2), verificou-se que a elevação do tempo de exposição das sementes aumentou as porcentagens de sementes mortas e de plântulas anormais, atingindo valores máximos aos 20 minutos de exposição. Este resultado mostrou que o tratamento térmico úmido pode prejudicar a qualidade fisiológica das sementes de A. mangium, sobretudo daquelas submetidas a períodos de exposição superiores a 15 minutos. Essa resposta pode ser atribuída, em parte, ao rompimento das biomembranas celulares ou desnaturação de proteínas dos tecidos embrionários devido ao calor (Machado, 2000), sendo considerados danos irreversíveis que podem reduzir tanto a germinação (Coutinho et al., 2007) quanto o potencial de armazenamento das sementes (Marcos Filho, 2015).

Como a termoterapia fundamenta-se nas diferenças de sensibilidade térmica existentes entre patógenos e sementes (Pereira et al., 2015), é necessário que os pontos letais para ambos sejam identificados. Neste estudo, os períodos de exposição de 10 e 15 minutos reduziram o percentual de ocorrência total dos fungos sem prejudicar a germinação das sementes sendo, portanto, considerado o tempo limite que as sementes de $A$. mangium podem permanecer expostas ao calor úmido à $70^{\circ} \mathrm{C}$, sem que sejam provocados danos à sua qualidade fisiológica. (a)

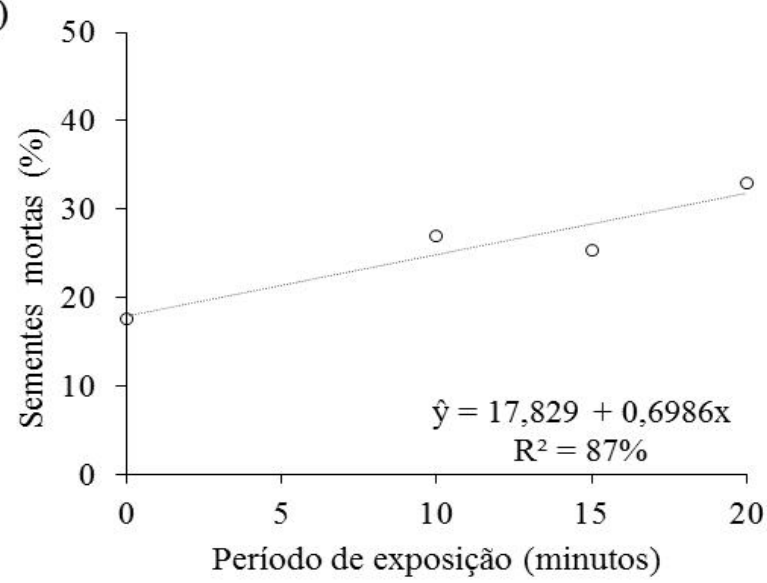

(b)

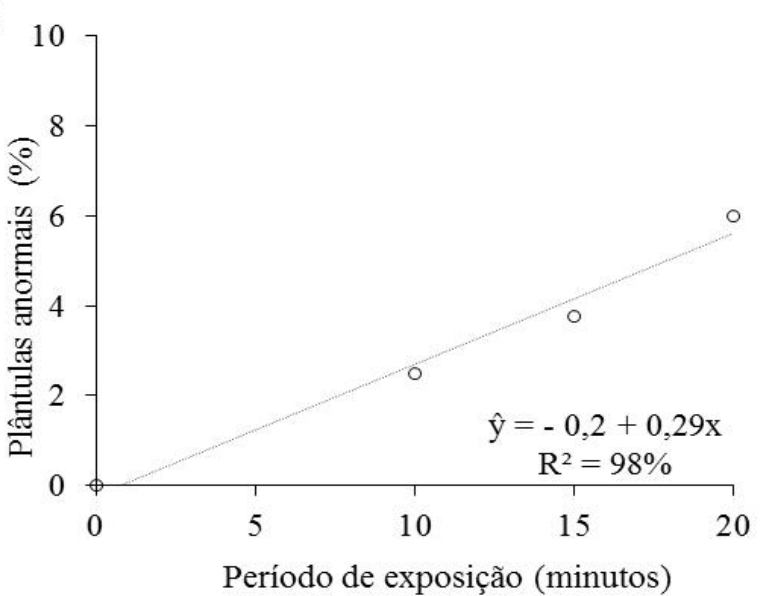

Figura 2 - Valores médios da porcentagem de sementes mortas (a) e plântulas anormais (b) de Acacia mangium submetidas ao tratamento térmico úmido $\left(70^{\circ} \mathrm{C}\right)$ durante 0 (controle), 10, 15 e 20 minutos. 


\section{CONCLUSÕES}

O tratamento térmico úmido à temperatura de $70^{\circ} \mathrm{C}$ durante 10 a 15 minutos supera a dormência das sementes de Acacia mangium e promove a redução da porcentagem de ocorrência dos fungos associados.

\section{AGRADECIMENTOS}

Ao Dr. Gualter Guenther Costa da Silva, professor da Unidade Acadêmica Especializada em Ciências Agrárias/Universidade Federal do Rio Grande do Norte pela doação das sementes e aos estagiários do Laboratório de sementes Florestais da mesma instituição pela coleta e beneficiamento das mesmas.

\section{REFERÊNCIAS BIBLIOGRÁFICAS}

Agustí-Brisach, C.; Pérez-Sierra, A.; Armengol, J.; García-Jiménez, J. \& Berbegal, M. (2012) - Efficacy of hot water treatment to reduce the incidence of Fusarium circinatum on Pinus radiata seeds. Forestry: An International Journal of Forest Research, vol. 85, n. 5, p.629-635. https://doi.org/10.1093/forestry/cps074

Barbosa, R.M.; Vieira, B.G.T.L.; Martins, C.C. \& Vieira, R.D. (2014) - Qualidade fisiológica e sanitária de sementes de amendoim durante o processo de produção. Pesquisa Agropecuária Brasileira, vol. 49, n. 12, p. 977-985. http://dx.doi.org/10.1590/50100-204X2014001200008

Baskin, J.M.; Nam, X. \& Baskin, C.C. (1998) - A comparative study of seed dormancy on germination in an annual and perennial species of Senna (Fabaceae). Seed Science Research, vol. 8, n. 4, p. 501-512. https://doi. org $/ 10.1017 /$ S0960258500004475

Benetti, S.C.; Santos, A.F.; Medeiros, A.C.S. \& Jaccoud Filho, D.S. (2009) - Levantamento de fungos em sementes de cedro e avaliação da patogenicidade de Fusarium sp. e Pestalotia sp. Pesquisa Florestal Brasileira, n. 58, p. 81-85. https://doi.org/10.4336/2009.pfb.58.79

Bewley, J.D.; Bradford, K.; Hilhorst, H. \& Nonogaki, H. (2013) - Seeds: physiology of development, germination and dormancy. New York: Springer: ed.3, 392 p.

Braga, M.P.; Olinda, R.A.; Homma, S.K. \& Dias, C.T.S. (2010) - Relações entre tratamento térmico, germinação, vigor e sanidade de sementes de tomate. Revista Brasileira de Sementes, vol. 32, n. 1, p. 101-110. http://dx.doi. org/10.1590/S0101-31222010000100012

Brasil (2009) - Regras para Análise de Sementes. Brasília: MAPA/ACS, 399 p.

Brasil (2013) - Instruções para Análise de Sementes de Espécies Florestais. Brasília: MAPA/ACS, 98 p.

Coutinho, W.M.; Silva-Mann, R.; Vieira, M.G.G.C.; Machado, C.F. \& Machado, J.C. (2007) - Qualidade sanitária e fisiológica de sementes de milho submetidas à termoterapia e condicionamento fisiológico. Fitopatologia Brasileira, vol. 32, n. 6, p. 458-464. http://dx.doi.org/10.1590/S0100-41582007000600002

Françoso, C.F. \& Barbedo, C.J. (2014) - Tratamentos osmóticos e térmicos para controle de fungos em sementes de grumixameira (Eugenia brasiliensis Lam.) e pitangueira (Eugenia uniflora L.). Hoehnea, vol. 41, n. 4, p. 541-552. http://dx.doi.org/10.1590/2236-8906-30/2013

Galiana, A.; Balle, P.; N'Guessan Kanga, A. \& Domenach, A.M. (2002) - Nitrogen fixation estimated by ${ }^{15} \mathrm{~N}$ natural abundance method in Acacia mangium Willd. inoculated with Bradyrhizobium sp. end grown in silvicultural conditions. Soil Biology \& Biochemistry, vol. 34, n. 2, p. 251-262. https://doi.org/10.1016/S00380717(01)00179-1

Honório, I.C.G.; Gomes, J.A.O.; Parreiras, N.S.; Brandão, D.S. \& Martins, E.R. (2010) - Termoterapia no controle de fitopatógenos de sementes de fava-d'anta (Dimorphandra mollis Benth.). Enciclopédia Biosfera, vol. 6, n. 11, p. 1-6.

Lazarotto, M.; Mezzomo, R. Maciel, C.G.; Bovolini, M.P. \& Muniz, M.F.B. (2013) - Tratamento de sementes de canafístula via calor úmido. Amazonian Journal of Agricultural and Environmental Sciences, vol. 56, n. 3, p. 268-273. http://dx.doi.org/10.4322/rca.2013.038

Lazarotto, M.; Muniz, M.F.B. \& Santos, A.F. (2010) - Detecção, transmissão, patogenicidade e controle químico de fungos em sementes de paineira (Ceiba speciosa). Summa Phytopathologica, vol. 36, n. 2, p. 134139. http://dx.doi.org/10.1590/S0100-54052010000200005 
Machado, J.C. (2000) - Tratamento de sementes no controle de doenças. Lavras: Editora UFLA, 138 p.

Maciel, C.G.; Muniz, M.F.B.; Santos, A.F. \& Lazarotto, M. (2012) - Detecção, transmissão e patogenicidade de fungos em sementes de angico-vermelho (Parapiptadenia rigida). Summa Phytopathologica, vol. 38, n. 4, p. 323-328. http://dx.doi.org/10.1590/S0100-54052012000400009

Maguire, J.D. (1962) - Speed of germination: aid in selection and evaluation for seedling emergence and vigour. Crop Science, vol. 2, n. 2, p. 176-177.

Marchi, J.L.; Cicero, S.M. \& Gomes Junior, F.G. (2011) - Utilização da análise computadorizada de plântulas na avaliação do potencial fisiológico de sementes de amendoim tratadas com fungicida e inseticida. Revista Brasileira de Sementes, vol. 33, n. 4, p. 652-662. http://dx.doi.org/10.1590/S0101-31222011000400007

Marcos Filho, J. (2015) - Fisiologia de Sementes de Plantas Cultivadas. Piracicaba: FEALQ, 2 ed., 660 p.

McCartan, S.A.; Webber, J.F. \& Jinks, R.L. (2015) - Hot-water treatment as a possible method for eradicating Chalara fraxinea (Hymenoscyphus pseudoalbidus) infection from ash fruits (Fraxinus excelsior L.). Quarterly Journal of Forestry, vol. 109, n. 1, p. 18-23.

Menten, J.O. \& Moraes, M.H.D. (2010) - Tratamento de sementes: histórico, tipos, características e benefícios. Informativo ABRATES, vol. 20, n. 3, p. 52-53.

Oliveira, M.D.M.; Nascimento, L.C.; Gonçalves, E.D.; Alves, E.U.; Guedes, R.S. \& Silva Neto, J.J. (2011) Qualidade sanitária e fisiológica de sementes de Amburana cearensis A.C. Smith submetidas à termoterapia e tratamento químico. Acta Scientiarum Agronomy, vol. 33, n. 1, p. 45-50. http://dx.doi.org/10.4025/actasciagron. v33i1.5645

Pereira, R.B.; Silva, P.P.; Nascimento, W.M. e Pinheiro, J.B. (2015) - Tratamento de Sementes de Hortaliças. 1.ㄹ ed., Brasília: Embrapa hortaliças. 16 p.

Santana, D.G. \& Ranal, M.A. (2004) - Análise estatística. In: Ferreira, A.G. e Borguetti, F. (Org.) - Germinação: do básico ao aplicado. Porto Alegre: Artmed, p. 197-208.

Schneider, C.F.; Gusatto, F.C.; Malavasi, M.M.; Stangarlin, J.R. \& Malavasi, U.C. (2015) - Termoterapia na qualidade fisiológica e sanitária de sementes armazenadas de pinhão-manso. Semina: Ciências Agrárias, vol. 36, n. 1, p. 47-56. http://dx.doi.org/10.5433/1679-0359.2015v36n1p47

Silva, F.A.S. \& Azevedo, C.A.V. (2002) - Versão do programa computacional Assistat para o sistema operacional Windows. Revista Brasileira de Produtos Agroindustriais, vol.4, n. 1, p. 71-78. http://dx.doi. org/10.15871/1517-8595/rbpa.v4n1p71-78 